\title{
Mesoscale simulation of complex transport phenomena far from equilibrium
}

\author{
Dr. Sauro Succi \\ IIT, Center for Life Nanosciences@La Sapienza, Rome, IAC-CNR, Rome, Italy \\ and IACS Harvard, Cambridge USA
}

Abstract - We present the main ideas behind the mesoscale modelling of complex transport phenomena far from equilibrium, along with a discussion of a range of applications, ranging from droplet generation in microfluidic devices, to nanoscale catalytic reactors as well as flows around complex biological structures. 